\title{
Prosodic focus in Bangla: A psycholinguistic investigation of production and perception
}

\author{
Arunima Choudhury \& Elsi Kaiser \\ University of Southern California \\ arunimac@usc.edu
}

\subsection{Introduction}

Cross-linguistically, the category of focus is often classified into two broad types: (a) New information Focus, and (b) Contrastive/Corrective Focus (Chafe 1976). In addition, prior work has identified three main strategies of marking focus: (a) Positional focus marking/syntactic focus marking, where the focused constituent occurs in a particular syntactic position; (b) Morphological focus marking, where focus is signaled with a focus particles/morpheme; and (c) Prosodic focus marking, where focus marking using pitch accenting and other intonational cues. This paper investigates how and whether Bangla/Bengali, an Indo-Aryan language spoken in the eastern parts of the South Asian subcontinent (India and Bangladesh), differentiates between two type of focus, namely new information focus and contrastive focus.

\subsection{Focus in Bangla}

Bangla is a relatively free word order language with a canonical SOV order. Bangla uses all three focus marking strategies to indicate focus. New-information focus in Bangla is marked using a positional focus strategy where the focused constituent occurs in the immediately pre-verbal position, which is the default 'focus position' in Bangla. Both subject and object can be marked for newinformation focus at the immediate pre-verbal position. Contrastive focus, on the other hand, appears to be marked using three different strategies: (i) positional marking through location of the focus in the immediate pre-verbal position, (ii) morphological marking via the cliticization of the particle $-i$, and (ii) prosodic marking (Choudhury 2010). As a result the different available interpretations for SOV word order are:

$\mathrm{S}[\mathbf{O}]_{\text {foc }} \mathrm{V}$ - new-information focus or contrastive focus on object; subject is unfocused $[\mathrm{S}]_{\text {foc }} \mathrm{O} \mathrm{V}$ - new-information focus or contrastive focus on subject; object is unfocused [S O V $]_{\text {foc }}$ - Broad focus (whole sentence is new information)

Hayes \& Lahiri (1991) have shown that Bangla (standard Kolkata variety) employs $\mathrm{L}^{*}+\mathrm{H}_{\mathrm{P}}$ pitch contour to indicate, classifying new-information and contrastive focus in the same category (see also Khan to appear for a detailed analysis of Standard Bangladeshi Bengali).

\subsection{Our Study}

To broaden our understanding of the relationship between information-structure, prosody and syntax, we conducted a production study followed by a perception study investigating two main questions: (i) when all syntactic cues are removed and the word order is SOV, do Bangla speakers distinguish new-information vs. contrastive focus prosodically, and (ii) does the position of the focused constituent matter? In particular, are potential prosodic distinctions amplified in the default focus position?

\subsection{Production Study}

Keeping the word order constant (canonical SOV order), we manipulated focus-type (newinformation/contrastive focus) and the grammatical role of the focused constituent (subject/object). Therefore, the study had a 2x2 design with four conditions: (i) Subject new-information focus, (ii) subject contrastive focus, (iii) object new-information focus and (iv) Object contrastive focus. The production study used a question-answer paradigm: We used wh-questions to elicit new-information focus (ex.1a,b) and yes/no questions to elicit contrastive/corrective focus (ex.1c,d). Speakers saw 
question-answer pairs. They were asked to read the questions and to say the answer out loud (e.g. ex.2, 'Father bought a car.'). Five native speakers of Bangla participated. The study had 20 target trials and 32 filler trials.

(1)

a. Subject-wh (new-information)

Who bought a car?

baba gaRi kinlo

$\underline{\mathbf{S}} \mathrm{OV}$

b. Object-wh (new-information)

'Father bought a car'

What did father buy?

baba gaRi kinlo

'Father bought a car'

SQ

c. Subject-y/n (corrective)

Did neighbor buy a car?

baba gaRi kinlo

$\underline{\mathbf{S}} \mathrm{OV}$

d. Object-y/n (corrective)

'Father bought a car'

Did father buy a computer? baba gaRi kinlo

SQ

'Father bought a car'

(2) Answer (elicited from speakers in the production study):

baba gaRi kinlo

SOV

Father car bought

'Father bought a car'

\subsection{Perception Study}

Sentences recorded during the production study were used as stimuli for the perception study. The perception study was a forced-choice task: For each grammatical role (subject/object), listeners (12 native speakers of Bangla) saw a wh-question and a yes-no question on the screen (left/right positions were balanced) and heard a sound file elicited by a wh-question or a yes/no-question of the same grammatical role (shown in example 3a,3b). Participants were told to choose the question that the sound file was the most appropriate answer for, i.e. whether the sentence played in audio was an answer to a wh-question or a yes/no question. Since the word order of the auditorily-presented sentences was always SOV, then if listeners distinguish focus-types, we can attribute this to differences in the intonation/prosody. (All experimental materials were in Bangla.)

(2a) Screen showed two questions:

What did father buy?

Did father buy a computer? [Object Condition]

Participants heard:

baba gaRi kinlo

(2b) Screen showed two questions:

Who bought a car?

Father bought a car

(elicited by object wh-question or object y/n-question)

Participants heard:

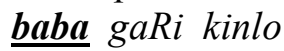

Did neighbor buy a car? [Subject Condition]

Father bought a car

(elicited by subject wh-question or subject y/n-question) 


\subsection{Results}

We analyzed the results in two possible ways. (i) Analysis 1: The percentage of match responses, that is, how often the question that participants choice matches the original context in which the sound file was elicited i.e. the expected question (as shown in Figure 1); and (ii) Analysis 2: The overall percentage of wh-question choices, that is, for each of the four conditions, how often did people choose the wh-question (as shown in Figure 2).

Figure 1: Percentage of match responses

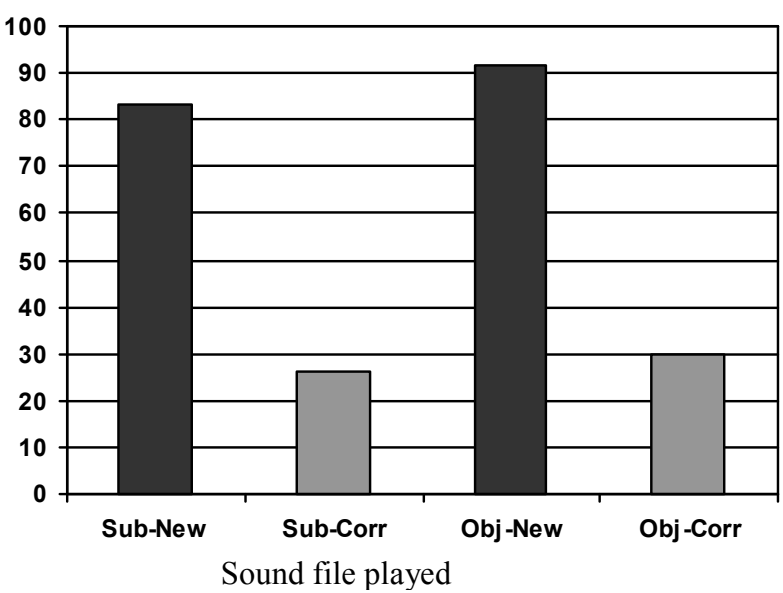

Figure 2: Percentage of wh-question choices

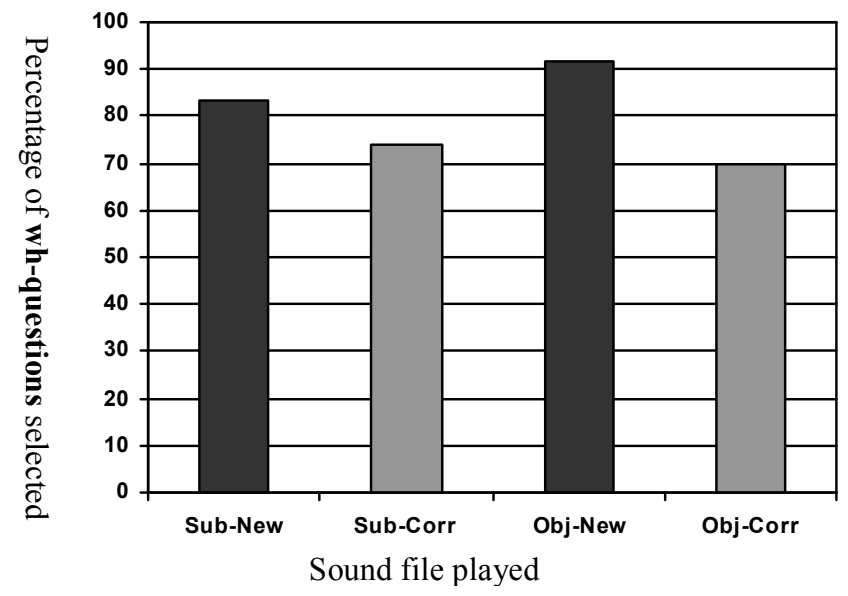

When we look at the rate of matching choices (Analysis 1), we see that new-information sound files elicited a higher rate of matching responses than contrastive focus sound files, for both subject and object focus. This shows that overall, participants tend to choose the wh-question over the yes/noquestion, i.e., there is a preference for the new-information focus type for both subject and object. However, to get a better sense of whether participants are able to perceive a difference between newinformation focus and contrastive focus, we graphed the same data in a different fashion, as shown in Figure 2: Here, the same data is shown in terms of the rate of wh-question choices. As expected, all bars are rather high, but now it is clearer that the preference for wh-questions is weakened when the sound file played was of contrastive focus type (black bars are taller than grey bars). Figure 2 shows that this weakening effect can be seen numerically for both subjects and objects. However, paired ttests show that while there is a significant decrease in the selection of wh-questions (in contrastive focus type conditions) in the object-focus conditions $(p<0.05)$, this effect is not significant in the subject-focus conditions $(p>0.3)$

\subsection{Perception Study Conclusions}

Participants show an overall preference for the new-information focus, as shown by the fairly high rate of wh-question choices in all conditions. However, when played a contrastive focus type target sentence, the proportion of wh-question choices was significantly weakened in the object-focus conditions. The subject-focus conditions showed only a numerical effect. This suggests that the participants are able to perceive the distinction between the two focus types using prosodic cues; however they are more efficient in distinguishing between the focus types for objects than for subjects. It seems that listeners are prosodically distinguishing between focus-types only when the focused constituent is an object, in the default focus position.

\subsection{Acoustic Analyses of Production Study}

We conducted acoustic analyses of the production study data to get more insight into the asymmetry observed in the perception of prosodic cues. We looked at pitch (f0), intensity and duration. We focus here on pitch (f0), as we did not find any significant effects of intensity and duration. Mean F0 
analyses were conducted on 10 time-normalized segments centered at noun offset using Prosody Pro. The pitch contours of the acoustic analyses are shown in Figure 3.

Figure 3. Plot of fundamental frequency (f0) of production study data

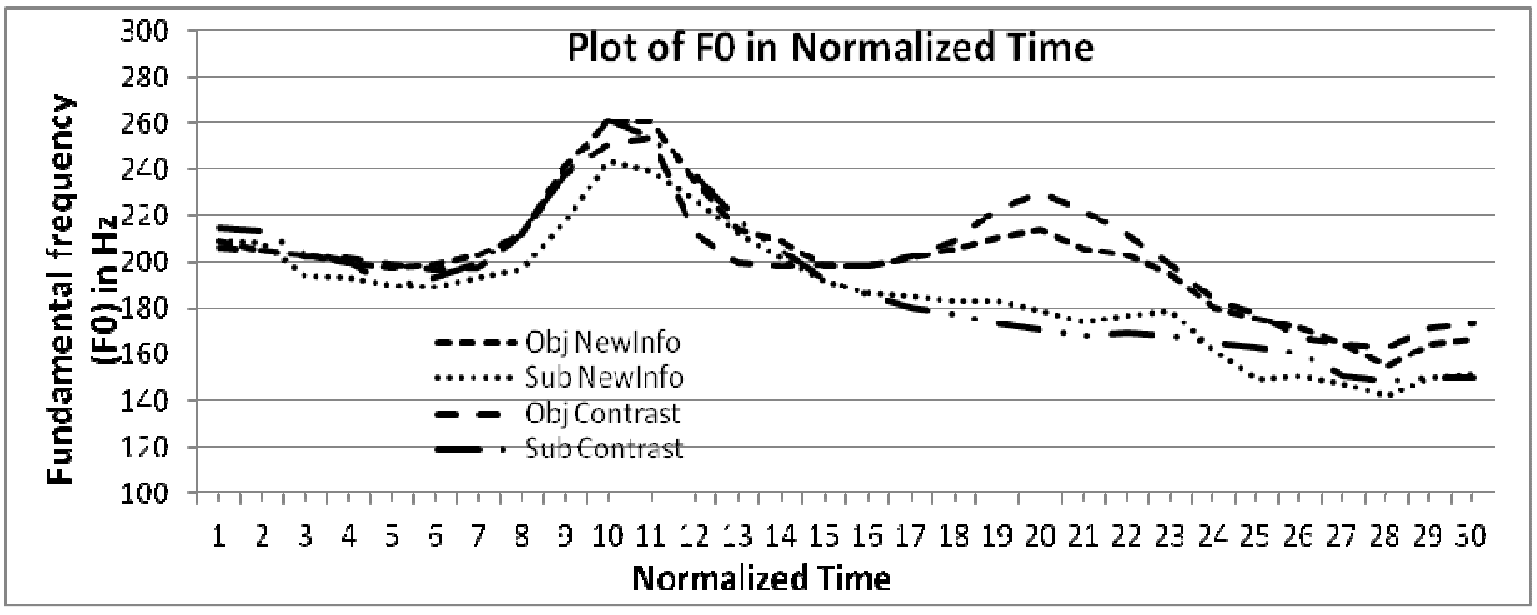

Time segments 1-10: subject, 10-20: object; 20-30: verb

The results show that new-information objects have lower mean F0 than contrastively-focused objects (p's<.05). In addition, focused objects have higher F0 than unaccented objects. Like objects, new-information subjects have lower F0 than contrastively-focused subjects (p's $<.05)$. Crucially, unaccented subjects do not differ significantly from contrastively-focused or new-information subjects. In general, subjects have high F0, unlike objects, presumably due to initial prominence. This suggests that the asymmetrical perceptibility of focus types for subjects vs. objects may be due to 'crowding' crowding of F0 on the subjects in all four conditions; therefore the high F0 in subjects is not a reliable cue for perceptibility. Alternatively, one can also say that the prosodic distinctions between the focus types is amplified at the default focus position. In future work, we plan to investigate the effects of linear position, syntactic position and grammatical role more closely, in order to better understand what underlies the asymmetry we observed between subjects and objects.

\subsection{General Conclusions}

The results of our production and perception studies showed that (i) Bangla, which is known to have positional and morphological focus marking, also uses prosodic cues to mark focus, and that (ii) the perceptibility of prosodic cues interacts with syntactic position: Differences between focus-types are clearest with focused constituents in default focus position. This suggests that the availability and the effect of prosodic cues for focus types interacts and varies with the syntactic position of the focused constituent.

\section{Selected References}

Bhattacharya, T (2000). "Bangla (Bengali)", in Gary, J. and Rubino. C., Encyclopedia of World's Languages: Past and Present (Facts About the World's Languages), WW Wilson, New York.

Choudhury, A (2010). A Study of Focus in Bangla, M.A. Thesis, U. Southern California. Hayes, B. \& A. Lahiri (1991). Bengali intonational phonology. Natural Language and Linguistic Theory. Khan (to appear). Intonational Phonology of Standard Bangladeshi Bengali. Prosodic Typology. ed. S.-A. Jun. Ladd, D.R. (1996). Intonational phonology. Cambridge Studies in Linguistics 79.

Pierrehumbert, J. (1980) The phonology and phonetics of English intonation. PhD thesis, MIT.

Simpson, A. \& J. Watkins (2006). Constituent Focus in Burmese: a Phonetic and Perceptual Study. (Vol. 27-

66). Studies in Burmese Linguistics/Pacific Linguistics. Canberra.

Watson, D.G, M. K. Tanenhaus \& C. A. Gunlogson (2008). Interpreting Pitch Accents in Online

Comprehension: $\mathrm{H}^{*}$ vs. $\mathrm{L}+\mathrm{H}^{*}$. Cognitive Science 32:1232-1244.

Xu, Y. (2011). Prosody Pro www.phon.ucl.ac.uk/home/yi/ProsodyPro/ 